\title{
CYCLE BIOLOGIQUE DE DIPLOTRIAENA SOKOLOWI SKRJABIN, 1916, FILAIRE OVIPARE PARASITE DU MARTIN-PECCHEUR HALCYON SENEGALENSIS AU TOGO
}

\author{
J.-C. QUENTIN*, C. SEUREAU** et S. D. KULO* \\ Collaboration technique : K. S. Agbalo* et A. Anglo**
}

RÉSUMÉ. Une Filaire ovipare parasite du Martin-pêcheur Halcyon senegalensis au Togo est identifiée à Diplotriaena sokolowi Skrjabin, 1916. L'évolution des trois premiers stades larvaires obtenue par l'infestation expérimentale de l'Orthoptère Acrididae Tylotropidius patagiatus Karsch, est décrite et figurée. La larve est infestante après 14 jours de développement à $30^{\circ} \mathrm{C}$. Ses structures céphaliques diffèrent de celles des autres troisièmes stades de Diplotriaenidae connus; elles récapitulent par contre les structures céphaliques adultes du genre Serratospiculum confirmant ainsi les affinités entre les Diplotriaeninae et les Dicheilonematinae.

Comme pour D. tridens et pour D. tricuspis, les larves de D. sokolowi s'encapsulent dans le tissu adipeux de l'Insecte. La réaction cellulaire d'encapsulement des Diplotriaenidae est comparée à la réaction cellulaire provoquée par les Spiruridae et les Onchocercidae dans le tissu adipeux de leur hôte intermédiaire.

\section{Life cycle of Diplotriaena sokolowi Skrjabin, 1916, an oviparous filarial Nematode parasite of the kingfisher Halcyon senegalensis in Togo.}

SUMMARY. An oviparous filarial nematode parasite of the kingfisher Halcyon senegalensis in Togo, is identified as Diplotriaena sokolowi Skrjabin, 1916. The development of the first three larval stages in experimentally infected Tylotropidius patagiatus Karsch (Orthoptera, Acrididae) is described and illustrated. Larvae are infective after 14 days of development at $30^{\circ} \mathrm{C}$. Their cephalic structures differ from those of the third-stage larvae of the known Diplotriaenidae; on the other hand, they recapitulate the adult cephalic structures of the genus Serratospiculum and confirm the affinity between the Diplotriaeninae and the Dicheilonematinae.

As in D. tridens and D. tricuspis, larvae of D. sokolowi are encapsulated in the insect adipose tissue. The cellular encapsulation in Diplotriaenidae is compared with that provoked by Spirurid and Onchocercid nematodes in the adipose tissue of their intermediate host.

\footnotetext{
* Laboratoire de Zoologie, École des Sciences, Université du Bénin, BP 1515 Lomé, Togo.

** Laboratoire d'Histophysiologie fondamentale et appliquée, ERA-CNRS 570, Université P. et M. Curie, 12 rue Cuvier, F 75005 Paris.

Accepté le I 5 mars 1982.
} 
Un Halcyon senegalensis capturé dans la localité d'Agomé Yoh près de Palimé, Togo, le 4 mars 1981, présente au niveau de la cavité péricardique et des sacs aériens 8 mâles et 10 femelles d'une Filaire ovipare. Il s'agit de l'espèce Diplotriaena sokolowi Skrjabin, 1916. Nous en confirmons l'identification par l'étude morphologique des mâles et des femelles et réalisons expérimentalement son cycle biologique afin de connaître son évolution larvaire et les caractères de sa larve infestante.

Les specimens sont enregistrés au M.N.H.N. de Paris sous le numéro Sf 326.

\section{Morphologie des adultes}

Trident céphalique à extrémité apicale arrondie, non dilatée; bras du trident légèrement inégaux en longueur, ornés de stries convergentes vers leur partie antérieure. Cuticule de la région apicale $(f i g .1 \mathrm{~A}$ ) présentant de courtes stries convergeant vers la bouche. Anneau nerveux et pore excréteur regroupés dans la région antérieure au niveau d'un court œsophage musculaire ( fig. $1 I, F$ ); œsophage glandulaire très allongé.

Mâle (fig. $1 \mathrm{D}, E, F, G, H$ ). Bourse caudale représentée sur les figures $1 \mathrm{G}$ et $1 \mathrm{H}$; spicule gauche rectiligne terminé par une pointe acérée, spicule droit vrillé deux fois sur lui-même, orné d'une aile latérale à partir de la première torsion. Papilles cloacales disposées sur la face ventrale en deux V emboîtés l'un dans l'autre à partir de la pointe caudale.

Dimensions relevées sur un mâle long de $2,8 \mathrm{~cm}$ : largeur $580 \mu$; longueur des tridents 170 à $200 \mu$; écart des tridents en vue apicale $80 \mu$, écart des pores amphidiaux IIo $\mu$, anneau nerveux et pore excréteur situés à 230 et $380 \mu$ de l'apex ; profondeur de la bouche $I_{5} \mu$, longueurs respectives de l'œsophage musculaire et de l'œsophage glandulaire 600 et $3100 \mu$; naissance du testicule à $600 \mu$ de l'apex, longueur du spicule gauche $740 \mu$ (760 sur un deuxième specimen), largeur 30 à $35 \mu$; longueur du spicule droit $525 \mu$ (560 sur le deuxième specimen), largeur 42 à $50 \mu$, longueur de la queue $60 \mu$.

Femelle (fig. I A, B, C, I, G). Longueur 4,4 à $4,9 \mathrm{~cm}$, largeur $650 \mu$; longueur des tridents 180 à $185 \mu$; longueur de chaque bras $112-145 \mu$, écart des tridents en vue apicale $84 \mu$, écart des pores amphidiaux I $24 \mu$; anneau nerveux et pore excréteur situés à 240 et $290 \mu$ de l'apex, profondeur de la bouche $20 \mu$, vagin ( fig. $I, I$ ) situé à $560 \mu$ de l'apex, dimensions des œufs $54-56 \mu \times$ $35 \mu$ (fig. $I, J)$.

\section{Identification}

Par les dimensions générales du corps et des organes, nos specimens se rapprochent de l'espèce Diplotriaena tridens Molin, 1858, qui présente une large répartition géographique.

Cependant, le sommet des tridents ne présente pas les deux sillons caractéristiques de D. tridens et par ailleurs, comme nous le constaterons dans la suite de ce travail, les larves infestantes du Diplotriaena du Martin-pêcheur sont différentes de celles de D. tridens. 

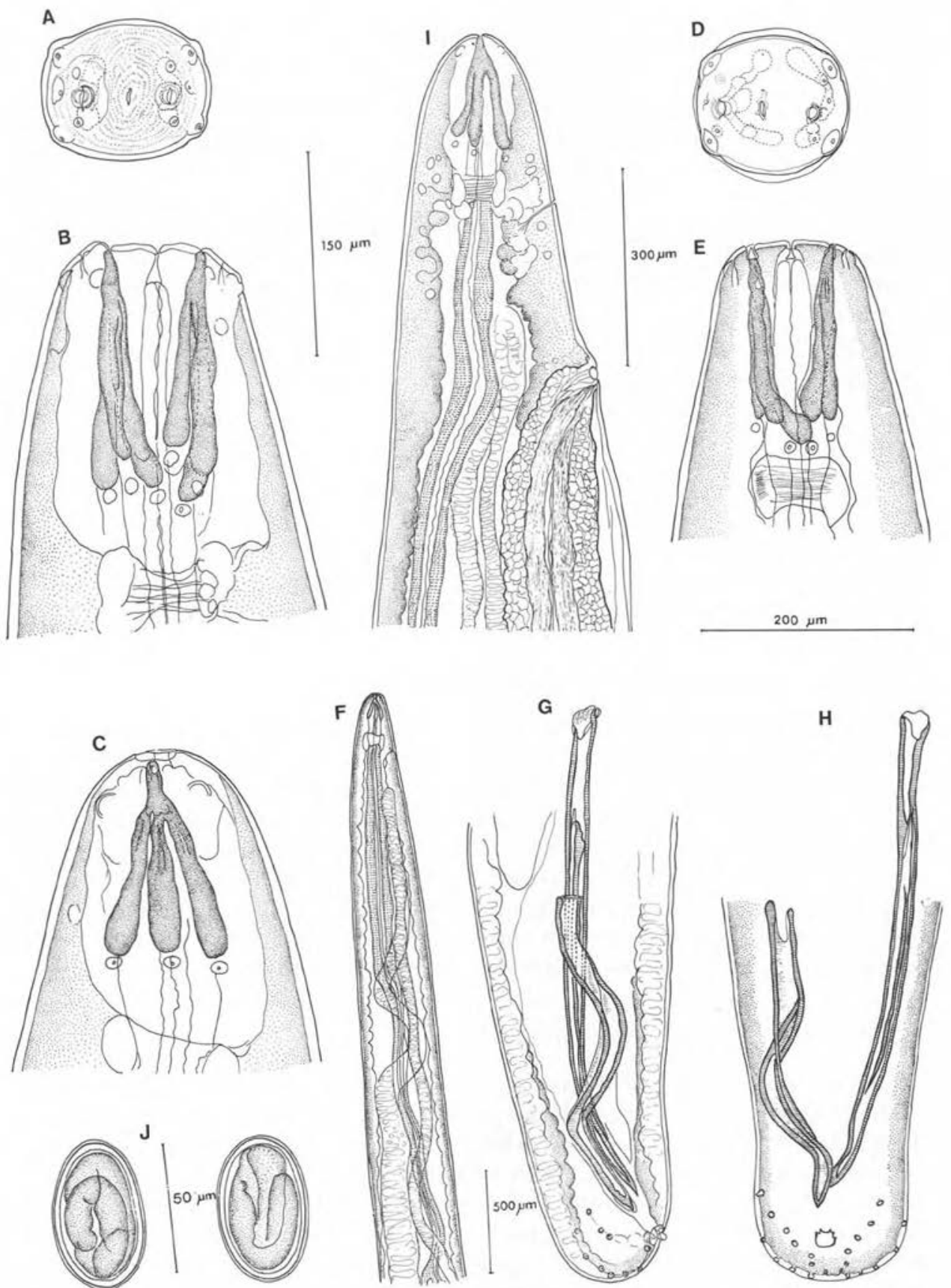

FIG. I. - Diplotriaena sokolowi, adultes mâle et femelle. A, B, et C : tête d'une femelle représentée respectivement en vue apicale, dorsale et latérale ; D et E : tête d'un mâle représenté respectivement en vue apicale et dorsale ; F : extrémité antérieure et région cesophagienne du mâle ; $\mathrm{G}$ et $\mathrm{H}$ : bourse caudale du mâle représentée successivement en vue latérale et ventrale : I : extrémité antérieure de la femelle, vue latérale de l'ovéjecteur ; J : œufs.

A, B et C : éch. I $50 \mu, \mathrm{D}$ et E : éch. $200 \mu, \mathrm{F}:$ éch. $500 \mu, \mathrm{G}, \mathrm{H}$ et I : éch. $300 \mu$, J : éch. $50 \mu$. 
Par contre, nos specimens s'apparentent étroitement, par l'aspect des tridents et la morphologie aciculée de l'extrémité terminale des spicules, à l'espèce $D$. sokolowi Skrjabin, 1916, redécrite par Anderson (1959), d'après des specimens récoltés par Chabaud et Rousselot (1955) chez Halcyon leucocephala et Halcyon malimbrimus en Afrique. Les longueurs plus importantes des spicules relevées sur notre matériel sont compatibles avec la grande taille de nos specimens. Elles sont en effet voisines des dimensions données par Skrjabin (1916) et Schmerling (1925) sur des individus de grande taille.

Nous identifions par conséquent le Diplotriaena du Martin-pêcheur Halcyon senegalensis au Togo à l'espèce $D$. sokolowi Skrjabin, 1916.

\section{Cycle biologique}

Les œufs embryonnés du parasite ( fig. $2 \mathrm{~A}$ ) sont placés entre les pièces buccales d'Orthoptères Acrididae appartenant à l'espèce Tylotropidius patagiatus Karsch, maintenus en élevage à une température de $30^{\circ} \mathrm{C}$.

\section{1 - Allure du développement. Chronologie des mues.}

Les larves du premier stade sont récoltées dans l'hémocoele des Insectes $24 \mathrm{~h}$ après l'infestation. Après 7 jours, une larve encapsulée dans le tissu adipeux est à la fin du premier stade, et présente un décollement de l'exuvie dans la région céphalique. Les larves âgées de 9 jours extraites de leur capsule sont au deuxième stade mais restent enfermées, au niveau de leur région postérieure, dans l'exuvie du premier stade. A 11 jours, la larve édifie les structures céphaliques et caudales du troisième stade, et la cuticule commence à se décoller à ces niveaux. A 14 jours, les larves encapsulées dans le tissu adipeux sont en train de muer ; certaines présentent un capuchon cuticulaire du deuxième stade déchiré dans la région céphalique.

\section{2 - Organogenèse et croissance larvaire.}

La larve âgée de $24 \mathrm{~h}$ de $D$. sokolowi est remarquable par sa grande taille $(198 \mu)$. Elle présente un fort crochet céphalique et quatorze rangées cervicales de fines épines. A $48 \mathrm{~h}$, la cellule R1, initiale du mésoderme, augmente considérablement de volume au point d'occuper toute la largeur du corps ( $f i g .2 C$ ); les cellules rectales s'épaississent. A 7 jours, deux catégories de larves sont récoltées. Les larves récoltées dans l'hémocoele (fig. $2 \mathrm{D}$ ) ne mesurent que $250 \mu$ de long et ne présentent que 4 cellules mésodermiques. Par contre, les larves encapsulées dans le tissu adipeux ( fig. $2 \mathrm{E}$ ) mesurent $340 \mu$ et ont constitué leurs cordons mésodermiques; l'ébauche génitale est alors située en arrière de la cellule excrétrice. La larve du deuxième stade âgée de 9 jours mesure $500 \mu$ de long, elle atteint $640 \mu$ au $11^{\mathrm{e}}$ jour. Elle est caractérisée par la disposition très antérieure de l'ébauche génitale, par l'allongement de l'œsophage 


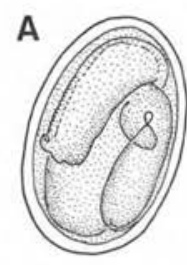

B
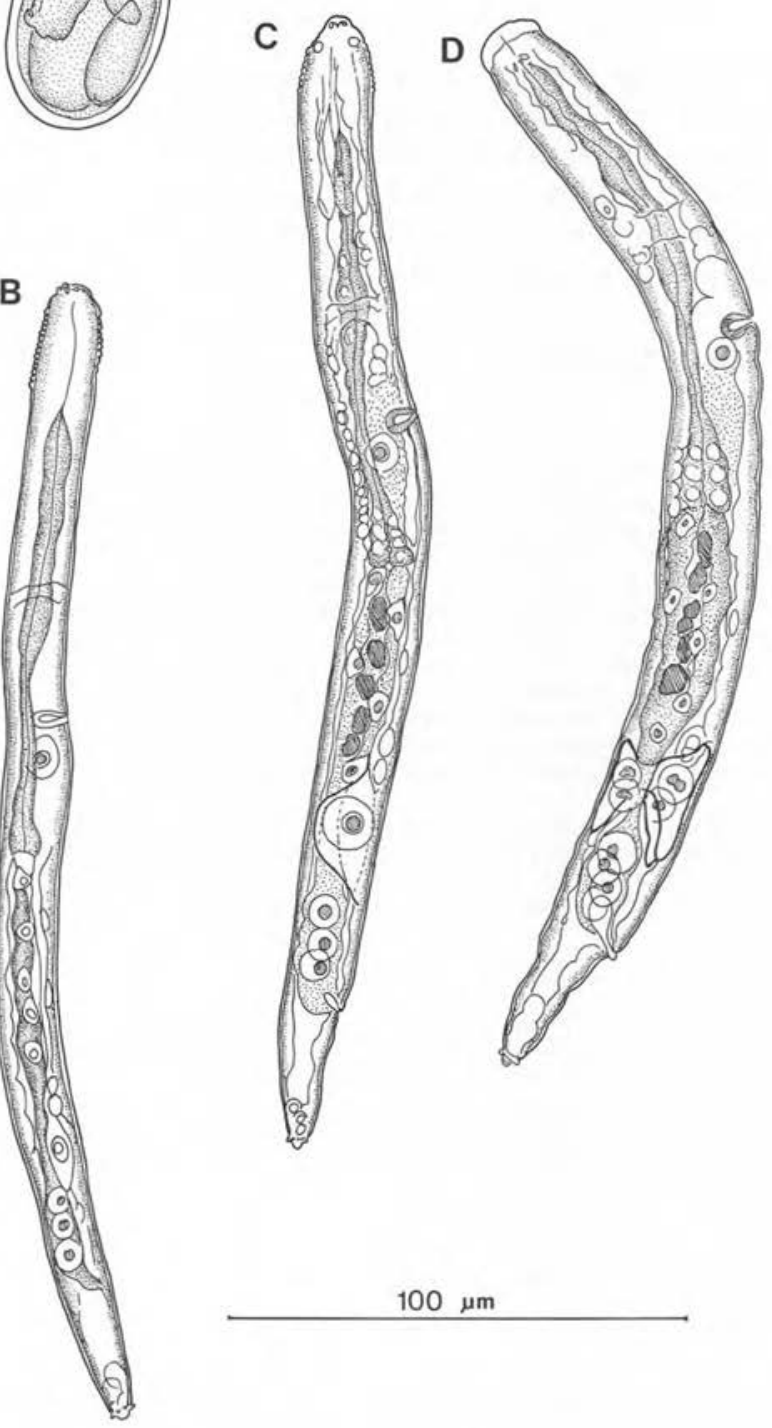

E
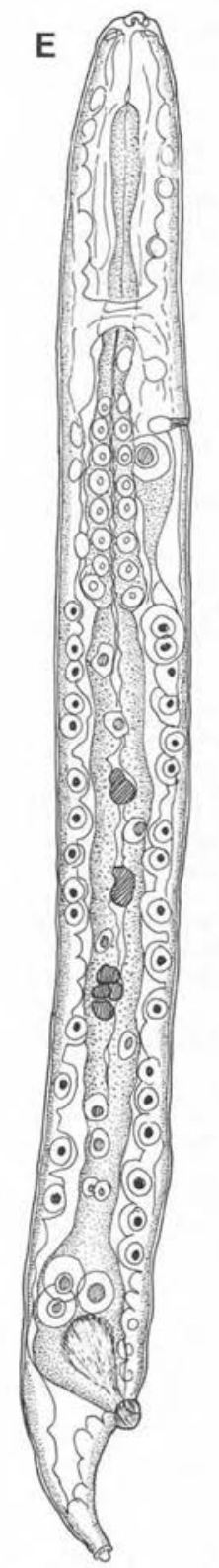

FIG. 2. - Diplotriaena sokolowi, premier stade larvaire. A : œuf embryonné ; B : larve après environ $24 \mathrm{~h}$ de développement chez l'hôte intermédiaire ; $\mathrm{C}$ : larve âgée de 2 jours ; D et E : larves âgées de 7 jours montrant deux niveaux de développement différents.
A, B, C, D et E : éch. roo $\mu$. 
et de l'intestin. Ce dernier ne renferme qu'un faible nombre de noyaux évalués à une quinzaine. Le "plug " secrété dès la fin du premier stade devient au 11 e jour un énorme bouchon de $45 \mu$ de long qui obture toute la cavité rectale et fait saillie à l'extérieur (fig. $3 \mathrm{~B}$ ).

Les larves de troisième stade récoltées le $14 \mathrm{e}$ jour mesurent $680 \mu$ de long. La croissance semble se poursuivre faiblement ; au $22^{\mathrm{e}}$ jour du développement, les larves infestantes mesurent $750 \mu$ de long sur 45-48 $\mu$ de large. Ces larves, courbées sur leur face dorsale, sont caractérisées par des structures céphaliques cuticulaires différenciées, par la position très antérieure de l'ébauche génitale et par le brutal tassement de l'intestin replié sur lui-même dans la partie terminale du corps.

\section{Dimensions des larves et de leurs organes.}

Premier stade larvaire (fig. 2):

- larve âgée de $24 \mathrm{~h}$ ( fig. $2, B$ ). Longueur $\mathrm{I} 98 \mu$, largeur $\mathrm{I} 4 \mu$, anneau nerveux et pore excréteur situés à 67 et $87 \mu$ de l'apex. Longueurs respectives du protorhabdion, de l'œesophage et de l'intestin : $27 \mu, 97$ et $74 \mu$; ébauche génitale et cellule RI situés à $68 \mu$ et 6 o $\mu$ de la pointe caudale, longueur des cellules rectales $26 \mu$, longueur de la queue $30 \mu$.

- larve âgée de $48 \mathrm{~h}$ (fig. 2, C). Longueur $250 \mu$, largeur $17 \mu$, anneau nerveux et pore excréteur situés à 64 et $87 \mu$ de l'apex. Longueurs respectives du protorhabdion, de l'œesophage et de l'intestin 30,95 et $67 \mu$; ébauche génitale et cellule $\mathrm{R}_{\mathrm{I}}$ situées à 87 et $72 \mu$ de la pointe caudale, longueur des cellules rectales $27 \mu$, longueur de la queue $32 \mu$.

- larve âgée de 7 jours, dans l'hémocoele ; stade 4 cellules mésodermiques (fig. 2, D). Longueur $250 \mu$, largeur $21 \mu$, anneau nerveux et pore excréteur situés à 56 et $85 \mu$ de l'apex. Longueurs respectives du protorhabdion, de l'œesophage et de l'intestin 10, 107 et 70 $\mu$; ébauche génitale et cellule RI situées à 80 et $70 \mu$ de la pointe caudale, longueur des cellules rectales $30 \mu$, longueur de la queue $34 \mu$.

- larve âgée de 7 jours encapsulée dans le tissu adipeux (fig. 2, E). Longueur 340 . largeur $30 \mu$, anneau nerveux et pore excréteur situés à 66 et $90 \mu$ de l'apex. Longueurs respectives du protorhabdion, de l'œsophage et de l'intestin 16, I 12 et $1_{42} \mu$, longueur des cellules rectales $40 \mu$, ébauche génitale situés à $135 \mu$ de l'apex, longueur de la queue $32 \mu$.

Deuxième stade larvaire :

- larve âgée de 9 jours $(f i g$. 3, A). Longueur $500 \mu$, largeur $37 \mu$, anneau nerveux et pore excréteur situés à $5^{8}$ et $95 \mu$ de l'apex; capsule buccale profonde de $5 \mu$, longueurs de l'œesophage et de l'intestin I 86 et $230 \mu$, longueur du rectum $44 \mu$, longueur de la queue $32 \mu$.

- larve âgée de 1 I jours ( $\mathrm{fig} ; 3, \mathrm{~B}$ ). Longueur $640 \mu$, largeur $40 \mu$, anneau nerveux et pore excréteur situés à 68 et roo $\mu$ de l'apex; longueurs respectives du pharynx, de l'cesophage et de l'intestin 12,295 et $240 \mu$, longueur du rectum $63 \mu$, longueur du " plug " $45 \mu$, ébauche génitale située à $124 \mu$ de l'apex, longueur de la queue $27 \mu$.

\section{3 - Morphologie et dimensions de la larve infestante.}

(fig. $3 \mathrm{D}, \mathrm{E}, \mathrm{F}$ et fig. 4 ).

De profondes modifications séparent cette larve du deuxième stade larvaire. Elles concernent essentiellement les structures céphaliques et la portion intestinale.

Les structures céphaliques ( $f$ g. $4 C, D, E$ et $F$ ). La bouche hexagonale ou arrondie est bordée d'un cadre buccal quadrangulaire renforcé régulièrement aux quatre coins d'une pointe cuticulaire dressée. Sur les deux côtés latéraux du cadre buccal, la zone péribuccale est renforcée de quatre plaques sous-cuticulaires jointives disposées en 

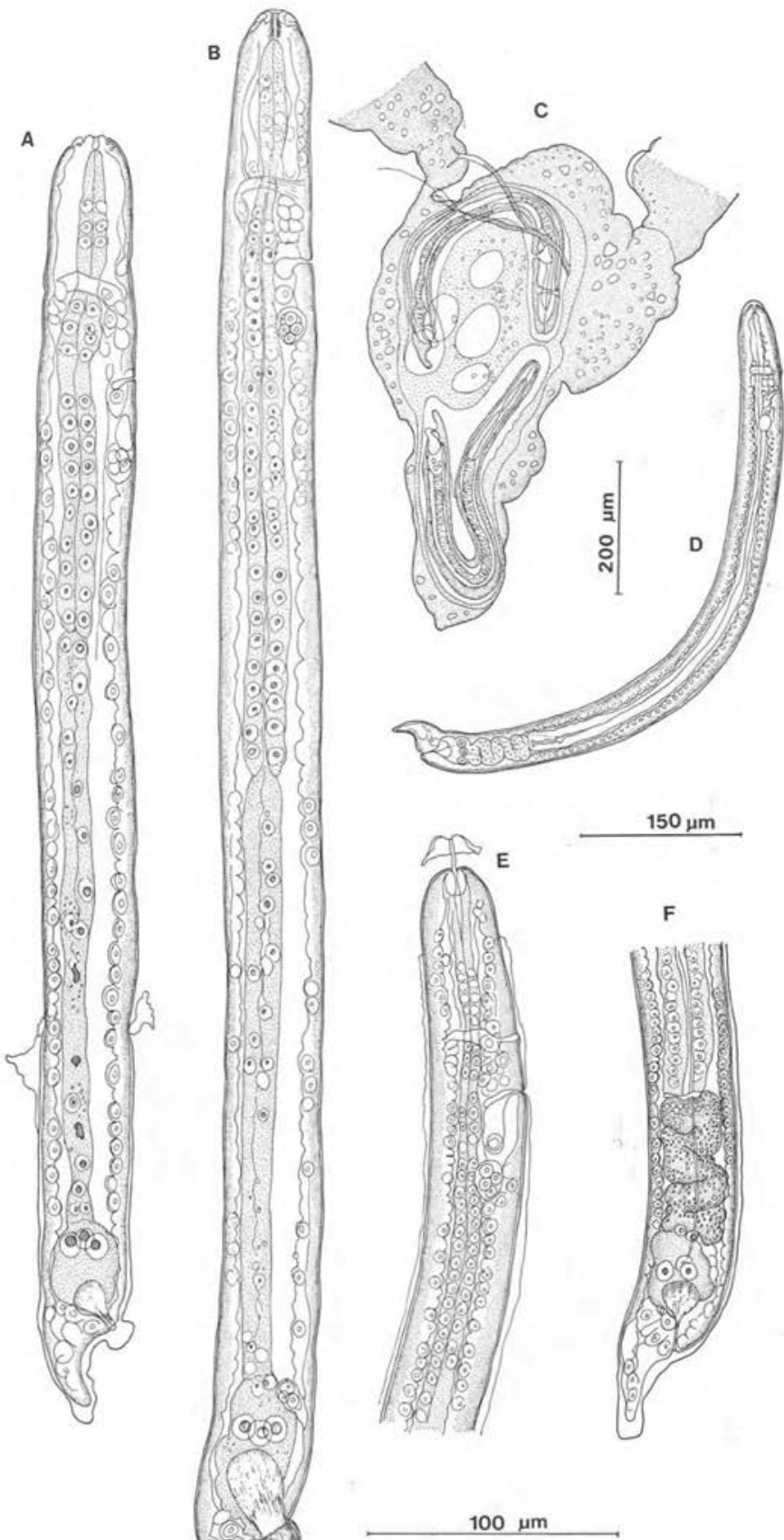

FIG. 3. - Diplotriaena sokolowi, deuxième stade larvaire et début du troisième stade. A : larve âgée de 9 jours dans l'exuvie déchirée du premier stade; B : larve de i I jours montrant un décollement de l'exuvie au niveau de la tête et de la pointe caudale ; C : larves du second stade II jours après l'infestation, encapsulées à l'intérieur du tissu adipeux de l'Insecte. Noter la minceur de la paroi de la capsule. D : larve au début du troisième stade, I4 jours après l'infestation, libérée de sa capsule, vue latérale; E et F : détails des régions œesophagienne et intestinale de cette larve, non encore dégagées de l'exuvie du $2^{\mathrm{e}}$ stade larvaire.

A, B, E et F : éch. roo $\mu, \mathrm{C}$ : éch. $200 \mu, \mathrm{D}:$ éch. $\mathrm{r}_{5} 0 \mu$. 

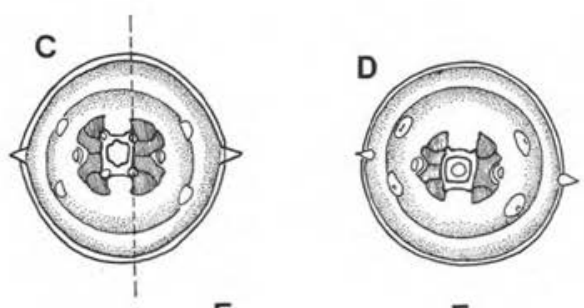

$\mathrm{E}$
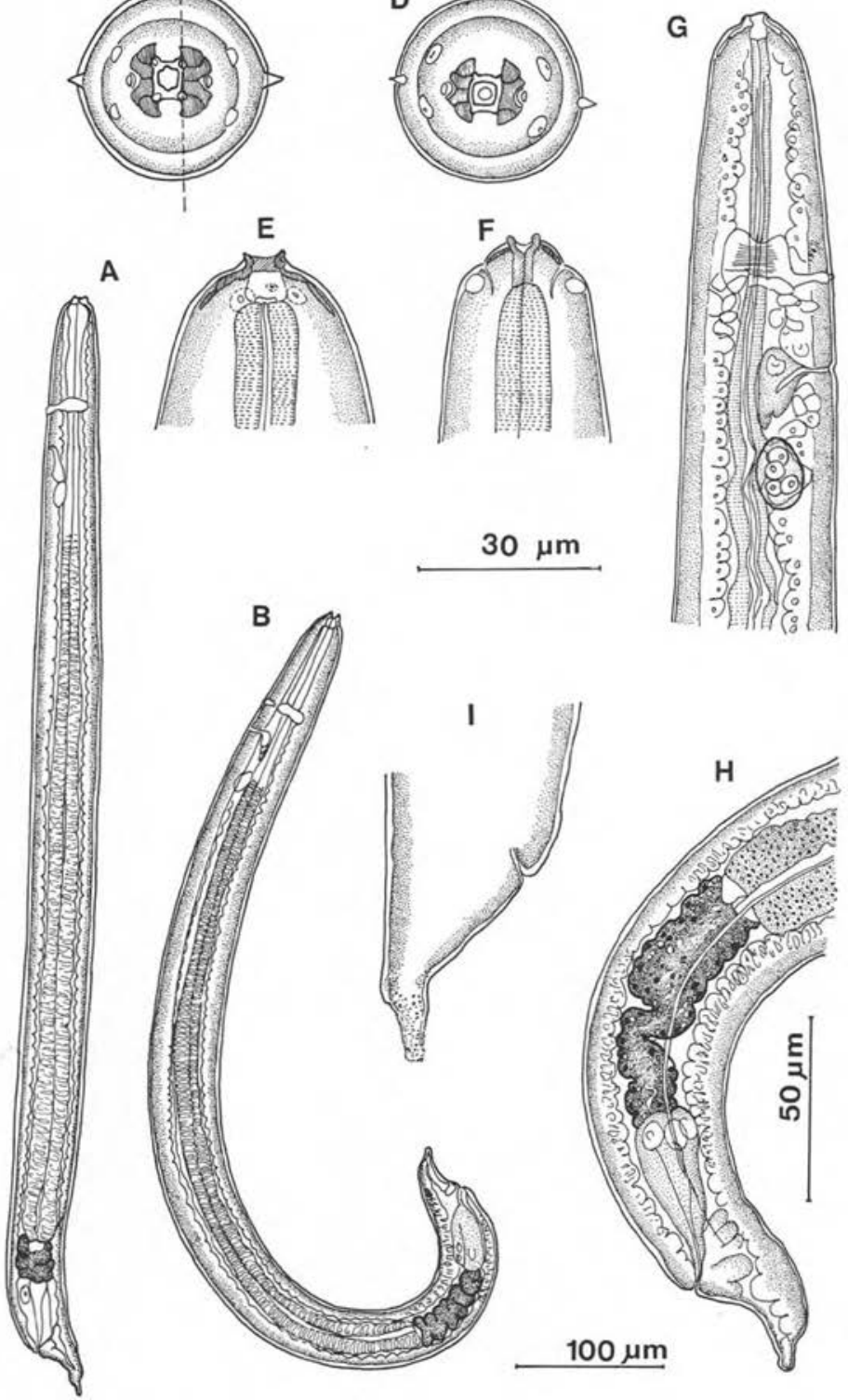

$30 \mu \mathrm{m}$

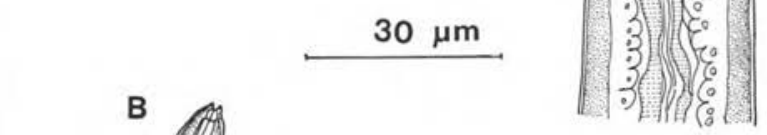

FIG. 4. - Diplotriaena sokolowi, troisième stade larvaire. A et B : larves 22 jours après l'infestation, vue latérale; C et D : deux vues apicales montrant le cadre péribuccal pourvu de quatre pointes, ainsi que les plaques sous-cuticulaires latérales au cadre buccal. Le tracé en pointillé indique le niveau de la coupe optique. $\mathrm{E}$ : coupe optique de la tête en vue latérale, passant au niveau du rebord et des pointes latérales du cadre buccal, les plaques péribuccales sont prolongées en profondeur par une lame sclérifiée non visible en vue apicale ; F : coupe optique en vue dorsale passant au niveau des amphides; G : extrémité antérieure en vue latérale, noter la position très antérieure de l'ébauche génitale; en arrière de la cellule excrétrice ; $\mathrm{H}$ : région intestinale et caudale; I : détail de l'ornementation de la pointe caudale pourvue de minuscules stries et aspérités cuticulaires.

A et B : éch. Ioo $\mu, C, D, E, F$, et I : éch. $30 \mu, \mathrm{G}$ et $\mathrm{H}:$ éch. $50 \mu$. 
éventail ${ }^{1}$. Les plaques internes sont faiblement écartées au niveau des amphides; les plaques externes sont prolongées en profondeur par une lame sclérifiée plus mince, tangente à la paroi, et de ce fait non visible en vue apicale ( fig. 2 E). Quatre papilles submédianes sont difficilement visibles à l'extrémité des plaques internes. Quatre papilles céphaliques sont rapprochées latéralement.

Cette larve présente également deux ailes latérales qui naissent en avant de l'anneau nerveux et s'estompent au niveau du rectum, ainsi qu'un intestin brutalement tassé sur lui-même et une pointe caudale rétrécie, ornée de fines aspérités cuticulaires (fig. 4I).

Dimensions d'une larve âgée de $r_{4}$ jours, au moment de la $2^{\mathrm{e}}$ mue : longueur $68 \mathrm{o} \mu$, largeur $45 \mu$, anneau nerveux et pore excréteur situés à 65 et $90 \mu$ de l'apex, profondeur de la capsule buccale ro $\mu$, longueur de l'œsophage $55^{\circ} \mu$, longueur occupée par l'intestin $54 \mu$, longueur du rectum $45 \mu$, ébauche génitale située à I $20 \mu$ de l'apex ; longueur de la queue $35 \mu$.

- larves âgées de 22 jours : longueur $750 \mu$, largeur $45 \mu$, anneau nerveux et pore excréteur situés à 72 et $105 \mu$ de l'apex, longueurs respectives de l'œsophage musculaire, de l'œsophage glandulaire et de l'intestin 150,500 et $30 \mu$, longueur du rectum $40 \mu$, ébauche génitale située à I $40 \mu$ de l'apex, longueur de la queue $40 \mu$.

\section{4 - Encapsulement cellulaire des larves infestantes chez l'Insecte.}

L'examen histologique de Criquets parasités montre que les larves infestantes de Diplotriaena sokolowi sont situées dans les lobes du tissu adipeux. Elles y provoquent une réaction cellulaire des adipocytes donnant naissance à une capsule dont le diamètre moyen varie entre 150 et $200 \mu$.

Cette capsule peut être de forme arrondie ou aplatie. Dans le premier cas (fig. $5 \mathrm{~A}$ ), une couche externe mince fibreuse et dense entoure une masse interne plus claire dans laquelle est logé le Nématode. A la limite entre ces deux zones, on observe des noyaux de taille légèrement supérieure à celle des noyaux des adipocytes environnants. Dans le second cas (fig. $5 \mathrm{~B}$ ), la réaction cellulaire d'encapsulement est plus discrète tant en ce qui concerne la paroi mince de la capsule, que les noyaux, peu différents de ceux des adipocytes.

Quelle que soit l'importance de la réaction cellulaire à la présence des parasites, ceux-ci se trouvent toujours isolés de l'hémocoele par une enveloppe provenant d'un lobe de tissu adipeux modifié. Aucune accumulation d'hémocytes ou de pigment n'a été observée en relation avec ces phénomènes d'encapsulement cellulaire.

\section{Discussion}

Les structures larvaires de $D$. solokowi sont différentes de celles des cinq Diplotriaenidae dont les cycles sont connus.

I. Les curieuses structures céphaliques munies d'un cadre surélevé de pointes et bordé de plaques sous-cuticulaires péribuccales de ce stade infestant de Diplotriaena s'observent également chez les stades infestants de Rictulariidae Pterygodermatites taterilli et $P$. affinis (cf. Quentin I970, Quentin et coll. 1976). Ces structures semblent apparaitre chez des larves dont les adultes ont des structures céphaliques très différenciées : tridents de Diplotriaena, capsule buccale complexe des Rictulariidae $P$. taterilli et P. affinis. 

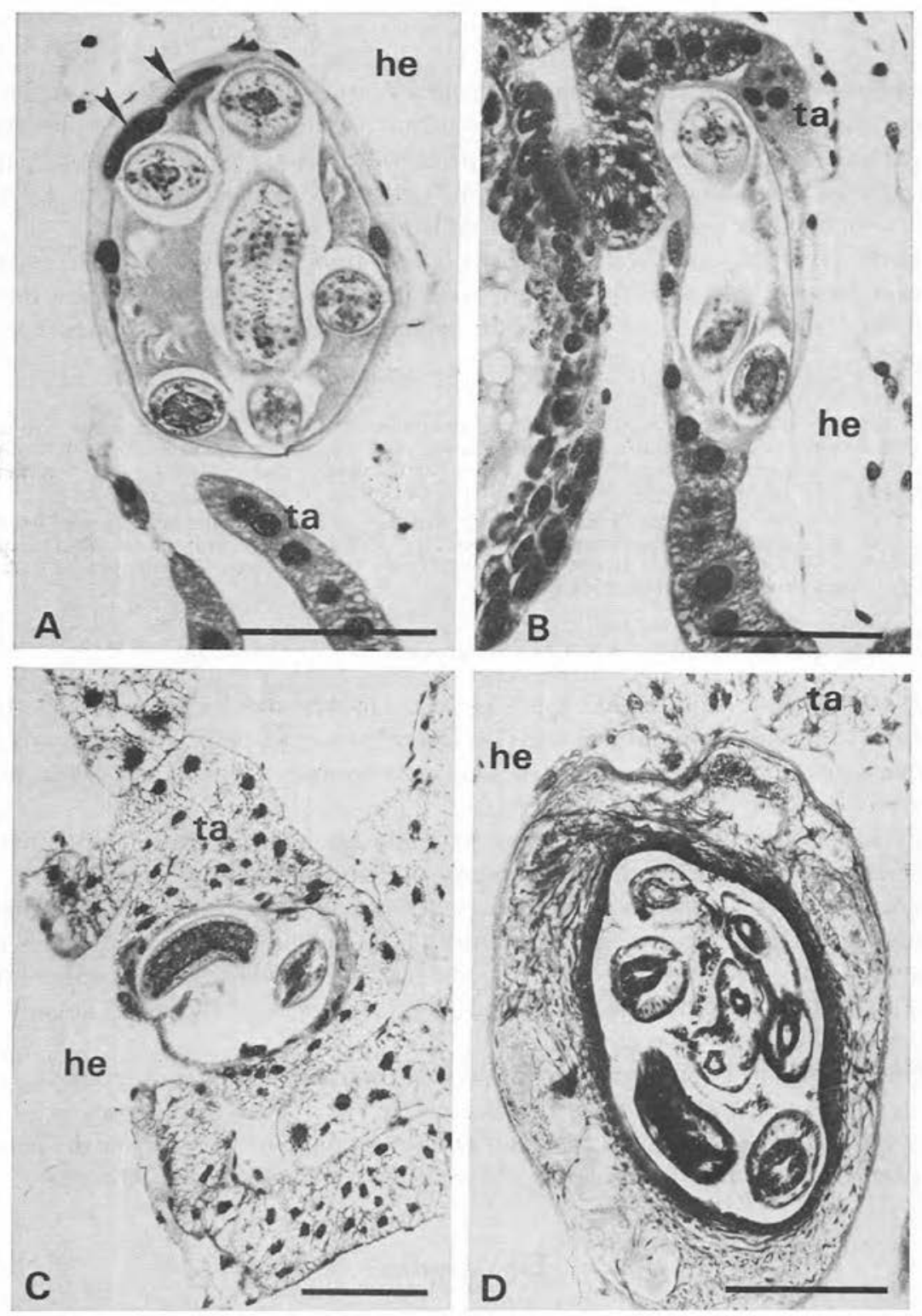

FIG. 5. - Encapsulement cellulaire des larves infestantes chez l'Insecte. A et B : Diplotriaena sokolowi chez Tylotropidius patagiatus, 22 jours après l'infestation expérimentale. En A, capsule de forme arrondie ; une mince couche externe entoure une masse non structurée où est logé le parasite; des noyaux de la capsule (flèches) sont légèrement hypertrophiés par rapport à ceux des adipocytes voisins. En B, la réaction cellulaire du tissu adipeux est discrète. C : Diplotriaena tridens chez Locusta migratoria, 40 jours après l'infestation expérimentale. Noter la faible réaction du tissu adipeux parasité. D : Protospirura muricola (Spiruridae) chez Locusta migratoria, 20 jours après l'infestation expérimentale. La capsule, de grande taille, possède une paroi fibreuse montrant une zone interne très dense entourant le Nématode.

ta : tissu adipeux, he : hémocoele. Trait d'échelle : roo $\mu$. 
Comparaison avec les structures larvaires des Diplotriaeninae:

- D. agelainus (Walton, 1927). Le cycle a été réalisé par Anderson (1957). La bouche est bordée également d'un cadre buccal muni de quatre pointes, mais l'ornementation cuticulaire péribuccale est constituée de six branches dont les deux médianes sont plus courtes et plus larges que les latérales. En outre, il existe quatre papilles submédianes proéminentes qui ne sont pas visibles sur notre matériel. La queue très courte présente une pointe effilée. La larve est de plus petite taille : $710 \mu$.

- D. bargusinica Skrjabin, 1917. Le cycle a été réalisé par Anderson (1962). La larve infestante est caractérisée par l'asymétrie ventro-dorsale des structures céphaliques ; le cadre cuticulaire péribuccal ne présente en effet de pointes que sur la face ventrale. Les quatre papilles submédianes sont bien visibles. Les larves infestantes sont petites (523-624 u).

- D. tridens (Molin, 1858). Bain et Vaucher (1973) ont montré que la région céphalique de la larve infestante est renforcée comme chez $D$. agelainus d'un anneau cuticulaire d'où partent six branches : quatre latérales et deux médianes plus larges, dont le contour correspond au tracé des fins cordons lobés entre les papilles et les amphides sur la cuticule céphalique des adultes de Psendaprocta. Les papilles submédianes ne sont pas visibles. Les larves infestantes sont trapues et mesurent $650 \mu$ de long sur 77 de large.

- D. tricuspis (Fedtchenko, 1874). Les larves infestantes obtenues par Cawthopn et Anderson (1980) présentent en plus des quatre papilles submédianes proéminentes, un cadre buccal hegaxonal et non quadrangulaire. Les plaques sous-cuticulaires péribuccales n'ont pas été observées. La longueur du corps est faible : $641 \pm 46 \mu$.

\section{Comparaison avec les structures larvaires des Dicheilonematinae:}

- Serratospiculum tendo (Nitzch, 1857). Le cycle réalisé par Bain et Vassiliades (1969) montre, chez la larve infestante, un anneau sclérifié hexagonal péribuccal hérissé de deux pointes médianes dont la forme est plus proche des Spirurides primitifs que des larves de Diplotriaeninae. Par ailleurs, la larve infestante est très allongée : $1090 \mu$, et présente à l'extrémité caudale une touffe de digitations.

\section{Comparaison avec les structures adultes de Dicheilonematinae:}

Les structures céphaliques larvaires en forme " d'épaulettes " sous-cuticulaires latérales de $D$. sokolowi sont comparables, par contre, aux structures céphaliques adultes de Serratospiculum qui sont munies de chaque côté de la bouche de plaques sclérifiées. Cette récapitulation ontogénique des structures céphaliques est un phénomène bien connu chez les Nématodes Spirurides. Elle permet dans le cas particulier de $D$. sokolowi, de situer cette espèce parmi les formes les plus primitives du genre Diplotriaena et de rechercher l'origine du genre Diplotriaena à partir d'une forme ancestrale proche du genre Serratospiculum.

Par sa localisation et par sa structure générale, la réaction cellulaire d'encapsulement de $D$. sokolowi chez l'Orthoptère Tylotropidius patagiatus est semblable à la réaction cellulaire d'encapsulement de D. tridens chez Locusta migratoria (fig. 5 C), 
comme à la capsule décrite par Cawthorn (1980) chez Melanoplus sanguinipes et Schistocerca gregaria parasités par D. tricuspis.

Cette première comparaison montre que les larves infestantes de ces trois Diplotriaena présentent une même localisation (dans le tissu adipeux de l'Insecte) et un même type de réaction cellulaire, qui caractérisent la Famille des Diplotriaenidae.

La comparaison de l'encapsulement larvaire des Diplotriaenidae avec les réactions histopathologiques provoquées également au niveau du tissu adipeux de l'hôte intermédiaire par les larves de Spiruridae et d'Onchocercidae, montre que le même tissu réagit différemment d'une Famille de Nématode à une autre. Dans le cas des Spiruridae ( fig. 5 D), la capsule est de grande taille (300 à $400 \mu$, le double de celle des Diplotrianidae), extrêmement fibreuse avec une couche interne de fibres plus denses entourant les parasites, et de grandes plages nucléaires provenant de l'hypertrophie des noyaux des adipocytes. Dans le cas des Onchocercidae, par contre, la réaction du tissu adipeux est particulièrement discrète puisque seules des modifications ultrastructurales des adipocytes parasités sont observées (Kan et Ho, 1972 ; Petit et SpitalierKaveh, 1979). Du point de vue des réactions cellulaires provoquées par les larves infestantes dans le tissu adipeux de l'hôte-Insecte, les Diplotriaenidae se situent donc entre les Spiruridae et les Onchocercidae.

Remerciements. Nous remercions vivement le Professeur Johnson, Recteur de l'Université du Bénin, pour les facilités qu'il a bien voulu nous accorder dans la réalisation de ce travail.

\section{BIBLIOGRAPHIE}

Anderson R. C. : Observations on the life cycle of Diplotriaenoides translucidus Anderson and members of the genus Diplotriaena. Can. J. Zool., 1957, 35, 15-24.

ANDERSON R. C. : Preliminary revision of the genus Diplotriaena Henry and Ozoux I9og (Diplotriaenidae: Diplotriaeninae). Parassitologia, 1959, I, 195-307.

ANDERSON R. C. : On the development, morphology and experimental transmission of Diplotriaena bargusinica (Filarioidea : Diplotriaenidae). Can. J. Zool., 1962, 40, I175-1186.

Bain O., Vassiliades G. : Cycle évolutif d'un Dicheilonematinae, Serratospiculum tendo, Filaire parasite du Faucon. Ann. Parasitol. Hum. Comp., I969, 44, 595-604.

Barn O., VAUCher C. : Développement larvaire de Diplotriaena tridens (Nematoda : Filarioidea) chez Locusta migratoria. Ann. Parasitol. Hum. Comp., 1973, 48, 81-89.

CAWTHORN R. J. : The cellular responses of migratory grasshoppers (Melanoplus sanguinipes F.) and African desert locusts (Schistocerca gregaria L.) to Diplotriaena tricuspis (Nematoda: Diplotriaenoidea). Can. J. Zool., I980, s8, ro9-1 13.

CAwThorn R. J., ANDerson R. C. : Development of Diplotriaena tricuspis (Nematoda : Diplotriaenoidea), a parasite of crows (Corvus brachyrhynchos Brehm), in intermediate and definitive hosts. Can. J. Zool., 1980, s8, 94-108.

Сhabaud A. G. : Remarques sur le cycle évolutif des Filaires du genre Diplotriaena et redescription de D. monticelliana (Stossich, 1890). Vie et Milieu, I956, 6, 342-347.

Chabaud A. G., Rousselot R. : Sur quelques Filaires d’Afrique équatoriale. Ann. Parasitol. Hum. Comp., 1955, 31, 53-98.

Kan S. P., Ho B. C. : Development of Breinlia sergenti in the fat-body of mosquitoes. II. Ultrastructural changes in the fat-body. $J$. Med. Entomol., 1972, 9, 255-261.

Petit G., Spitalier-Kaven H. : La Filaire Dipetalonema dessetae chez Aedes acgypti; étude du tissu adipeux parasité. Ann. Parasitol. Hum. Comp., 1979, 54, 81-92.

Quentin J. C. : Cycle biologique de Pterygodermatites (Mesopectines) taterilli (Baylis, 1928), Nematoda Rictulariidae. Ann. Parasitol. Hum. Comp., 1970, 45, 629-635. 
Quentin J. C., Seureau C., Vernet R. : Cycle biologique du Nématode Rictulaire Pterygodermatites (Multipectines) affinis (Jägerskiold, 1904). Ann. Parasitol. Hum. Comp., 1976, SI, $5 \mathrm{I}-64$.

Schmerling A. A. : Zur Kenntnis der Vogelfilarien (Squamofilaria n. gen. und Diplotriaena artemisiana n sp.) von Coracias garrula L. Centrabl. f. Bakt. Parasitenk. Infekt. Abt. II, 1925, 63. $267-27 \mathrm{r}$.

SKRJABIN K. I. : Parasitic Trematodes and Nematodes collected by the expeditions of Prof. V. Dogiel and I. Sokolov in British East Africa. Expedit. of Prof. V. Dogiel and I. Sokolov in British East Africa. Zoological Results, 1916, 4, Petrograd, I57 p. 